\title{
Impacto emocional y en calidad de vida de individuos afectados por amelogénesis imperfecta
}

\section{Dental and Emotional Perception in Individuals Affected by Amelogenesis Imperfecta}

\author{
Gina Murillo Knudsen DDS, MDSc ${ }^{1}$; Fabián Morales Cordero DDS2; \\ Luis Carlos Gamboa Chaves DDS; Ana María Meza Monge DDS³; Ana Cristina López Torres DDS³ \\ 1. Instructor Asociado, Facultad de Odontología, Universidad de Costa Rica, Costa Rica. \\ 2. Caja Costarricense de Seguro Social \\ 3. Odontólogo. Práctica privada. \\ Autor para correspondencia: Dra. Gina Murillo Knudsen - twingina24@hotmail.com \\ Recibido: 18-II-2015 \\ Aceptado: 18-II-2015 \\ Publicado Online First: 1-XII-2015 \\ D0I: http://dx.doi.org/10.15517/ijds.v0i0.22253
}

\section{RESUMEN}

Amelogénesis imperfecta (Al) describe defectos en el esmalte derivados de mutaciones genéticas que se manifiestan en anormalidades estructurales en la dentición temporal y permanente. A pesar del amplio conocimiento que aumenta día tras día a nivel mundial sobre las mutaciones genéticas asociadas a la Al, existe poca investigación en su efecto sobre la calidad de vida de los pacientes. El objetivo de este estudio es determinar, en un cohorte de pacientes costarricenses afectados por esta patología, la percepción emocional sobre su calidad de vida que esta condición ha generado. Miembros de varias familias portadoras de Al se entrevistaron y sus experiencias sirvieron de base para desarrollar un instrumento (cuestionario), el cual se utilizó para evaluar el impacto de este padecimiento, en su calidad de vida. Se incluyeron 18 personas afectadas de Al, provenientes de 17 familias. Los resultados más relevantes revelaron que un $100 \%$ de los participantes han sufrido burlas y rechazo; $77.8 \%$ se mostraron preocupados por la herencia a sus hijos; $66.7 \%$ les preocupa el costo del tratamiento; 89 $\%$ le atribuyen alta importancia a sus dientes, siendo no estadísticamente significativo por sexo ( $p=$ 0.732). El estudio indica que los profesionales en Odontología deben entender la Al no solo como un defecto en la estructura del esmalte dental, que demanda un manejo clínico especializado, sino como una condición que causa impacto negativo en la calidad de vida de quienes la padecen. Esto se debe considerar en la comunicación e interacción del profesional, los sujetos afectados y sus familias.

\section{PALABRAS CLAVE}

Amelogénesis imperfecta, Percepción emocional, Autoestima, Familias costarricenses.

MURILLO K., GAMBOA L., MORALES F., MEZA A., LÓPEZ A., 2015: Impacto emocional y en calidad de vida de individuos afectados por amelogénesis imperfecta.-ODOVTOS-Int. J. Dental Sc., 17-3 (September-December): 73-85. 


\section{ABSTRACT}

"Amelogenesis imperfecta" (Al) (prevalence up to 1 in 700) describes largely Mendelian enamel defects arising from gene mutations that present as structural abnormalities of the temporary and permanent teeth as a result of defective enamel formation. Al has a wide range of clinical presentations and phenotypes and affects both sexes. Despite increasing knowledge of the genetic mutations underlying $\mathrm{Al}$ there has been little research focussing on the effect of Al on the quality of life of $\mathrm{Al}$ patients. This study aimed to investigate the effects of Al on patient quality of life in a Costa Rican Al cohort. Affected family members were interviewed and their experiences used to develop an instrument (questionnaire) that was subsequently used to evaluate the impact of Al on their quality of life. $18 \mathrm{Al}$ patients from 17 families were included in the study. Our findings showed that $100 \%$ had been teased and had suffered social rejection; $77.8 \%$ were concerned about their children's inheritance and $66.7 \%$ were concerned by the cost of treatment; $89 \%$ placed a high importance on their teeth. Results were not statistically significant when sorted by $\operatorname{sex}(p=0.732)$. The results of this study indicate that dental professionals need to understand Al not only as defective tooth enamel structure demanding specialist clinical management but also the negative impacts of the condition on the lives of their patients. This should be taken in to account when communicating with those affected.

\section{KEYWORDS}

Amelogenesis imperfecta, Emotional perception, Self esteem, Costa Rican families.

\section{INTRODUCCIÓN}

\section{GENERALIDADES}

La amelogénesis imperfecta (Al) describe un grupo heterogéneo de defectos heredados en el esmalte heredados que reflejan el fracaso de la amelogénesis normal (1). Se refiere a un conjunto de trastornos de origen genético que producen alteraciones en el proceso de formación de los dientes temporales y permanentes y, en ocasiones, afecta otras estructuras orales y extraorales. Posee una prevalencia variable en la población mundial y afecta a ambos sexos (2).

El diagnóstico de la Al se centra en el patrón de herencia, el fenotipo y la relación con el tiempo de formación de la pieza dental (3). Además, involucra la historia familiar y el pedigrí (4).

La Al presenta distintos patrones hereditarios, dependiendo del gen que esté alterado, puede transmitirse a partir de un patrón autosómico, dominante 0 recesivo, 0 por medio de un patrón ligado al cromosoma $X$ (5). También puede originarse por medio de una mutación genética espontánea (6). Se caracteriza por tener su amplio rango de presentaciones clínicas (fenotipos) en donde se afecta la estructura y apariencia del esmalte de los dientes en ambas denticiones (7).

Según los autores Bonilla y Castro (2) y Kamala y Guddad (8) la Al presenta la siguiente clasificación:

- Hipoplásico: Su apariencia se debe a defectos en la fase secretora que conducen a volúmenes reducidos de proteína de la matriz del esmalte. Se presenta un esmalte muy delgado, ocasionando que la dentina se muestre a través de él e imparta un color amarillo-marrón al diente. El esmalte puede ser áspero o liso y brillante. Existe disminución del tamaño de la corona clínica y son frecuentes los diastemas, pérdida de dimensión vertical, así como también los problemas de 
sensibilidad en el paciente. Radiográficamente, se observa una forma cuadrada de las coronas y cúspides bajas 0 ausentes. La radiodensidad del esmalte se presenta normal.

- Hipomaduro: Se observa, clínicamente, un esmalte blando y rugoso, debido a que los cristales de hidroxiapatita se depositaron de manera adecuada, pero falló su proceso de maduración. Por el poco grado de dureza del esmalte, se producen microfracturas que favorecen la absorción de pigmentos exógenos, lo que origina coronas pigmentadas de color amarillo-café en combinación con un esmalte color blanco tiza. Radiológicamente, no se observa contraste entre la interfase esmaltedentina. Son comunes las facetas de desgaste por la disminución de la dureza del esmalte, al igual que problemas de sensibilidad, mordida abierta anterior o mordida profunda con pérdida de dimensión vertical. En rayos $X$, demuestra un espesor normal del esmalte, pero su densidad es igual a la de la dentina.

- Hipocalcificado: Es más común que la forma hipoplásica y similar a la hipomadura, ya que en ambas el esmalte presenta alteraciones en su dureza, la diferencia es la etapa de formación en que se presentó la mutación. Las coronas de los dientes son normales en tamaño y forma, debido a que el esmalte es de espesor regular y con pobre mineralización, este es suave y se fractura. Radiográficamente, el espesor del esmalte es normal, pero su densidad es incluso menor que la de la dentina.

- Hipomaduro-Hipocalcificado asociado a Taurodontismo: Esta es una combinación de ambas, con el peor pronóstico al combinar disminución en el grosor del esmalte con pérdida de dureza y cámaras pulpares grandes, lo cual aumenta la probabilidad de pulpitis (9).

Clínicamente, laAl se clasifica en dos grandes grupos: lesiones hipoplásicas e hipomineralizadas. El primer grupo se caracteriza por la presencia de piezas con zonas sin esmalte dental, mientras que el segundo presenta anatomía en apariencia normal, pero con una pobre mineralización del esmalte (10).

ENFERMEDADES CRÓNICAS Y SU IMPACTO PSICOLÓGICO Y EMOCIONAL EN LOS PACIENTES Y FAMILIARES

Aunque la Al no se cataloga como una enfermedad crónica se puede relacionar con múltiples aspectos, ya que quienes la padecen presentan características psicológicas muy similares a esta.

Coffield et al. (11) reportan que padecer Al presenta un marcado impacto en la salud psicológica de las personas afectadas comparable con el que generan condiciones sistémicas de salud, especialmente en edades tempranas.

De acuerdo con la OMS (12), la enfermedad crónica se define como un trastorno orgánico funcional que obliga a una modificación de los estilos de vida de una persona y que tiende a persistir a lo largo de su vida. Frente a la etiología no existe mayor claridad; existen una serie de factores que, en conjunto, dan pie a la enfermedad, entre estos el ambiente, los estilos de vida y hábitos, la herencia, niveles de estrés, calidad de vida y la presencia de una enfermedad persistente que puede pasar a ser crónica.

Una enfermedad crónica es un concepto que indica un curso prolongado de un padecimiento, que puede ser progresivo o letal y que requiere, en la mayoría de los casos, una atención médica continuada. Según la OMS (12), las enfermedades crónicas son de larga duración y, por lo general, de progresión lenta. La enfermedad crónica no desaparece, puede manifestar diferentes síntomas en función de la etiología, del curso, de la enfermedad y del pronóstico, entre las cuales se pueden mencionar: las enfermedades cardíacas y respiratorias, los infartos, el cáncer, la diabetes, 
epilepsia, fibrosis quística, distrofia muscular, autismo, esquizofrenia, alteraciones renales, paraplejía, por mencionar algunas.

Mercado y Hernández (13) señalan que las enfermedades crónicas han sido objeto de atención por parte de profesionales de la salud, quienes han realizado incontables estudios clínicos, bioquímicos, farmacológicos, epidemiológicos y, recientemente, desde la biología molecular; tal enfoque, sin embargo, no ha estado exento de críticas, algunas de ellas por excluir las perspectivas de actores como los enfermos y sus familiares en torno a su origen, su evolución y manejo, su desinterés por la dimensión subjetiva y experiencial del padecimiento.

Existen múltiples enfermedades crónicas que son poco frecuentes, lo que dificulta su estudio y manejo interdisciplinario. La mayoría requieren de una atención integral, incluyendo el abordaje psicológico. Aún existe mucho desconocimiento de estas enfermedades 0 condiciones, tanto en el diagnóstico, manejo, tratamiento como las adaptaciones que permitan a los individuos afectados normalizar sus necesidades en todos sus entornos (familiar, académico, social, laboral).

Vasermanas (14) dice que en esta interacción de distintos factores cobra relevancia el aspecto psicológico, relacionando el comportamiento de las personas afectadas con las respuestas del medio social en que se desenvuelven. Es por esto que es necesario conocer y comprender cómo se afrontan estas enfermedades para lograr ayudar y realizar un abordaje completo hacia los individuos afectados.

Gil-Roales (15) alude que la mayoría de las enfermedades crónicas afectan aspectos importantes de la vida en las personas que la padecen.

Asimismo, menciona la etapa crítica de la enfermedad crónica e identifica una serie de reacciones comunes en esta, las cuales están dirigidas a mantener un equilibrio emocionalmente razonable: mantenimiento o conservación de la autoimagen de forma satisfactoria, preservación de las relaciones familiares y sociales, y la preparación para el futuro. En esta fase, las personas asumen diferentes estrategias de afrontamiento, la más frecuente es la negación 0 minimización de la gravedad de la enfermedad y de sus consecuencias; otras estrategias frecuentes son buscar información acerca de la enfermedad y apoyo social y familiar.

Para Vinaccia y Orozco (16), una de los principales cambios, y quizás el que genera mayor deterioro, es el aspecto emocional, ya que la persona se ve obligada, necesariamente, a un proceso de adaptación rápido para el cual atraviesa por diferentes etapas que suscitan una serie de emociones comúnmente negativas como miedo, ira y ansiedad.

El sentimiento de miedo se presenta a 10 largo de la enfermedad. Así, cada recaída, visita médica y análisis frecuentes pueden convertirse en una gran fuente de temor ante la posibilidad de agravamiento. Después de un lapso, el paciente comprende las implicaciones que esta tiene; en ese momento aparecen los sentimientos depresivos, luego de entender las consecuencias y condiciones de padecer una enfermedad crónica. Estas reacciones emocionales no se presentan en todos los pacientes con padecimientos crónicos y su orden de aparición no es el mismo en todos los casos, incluso algunos no se manifiestan. Las reacciones emocionales asociadas no aparecen en una secuencia previamente establecida, pueden aparecer en el individuo en cualquier momento de la enfermedad o del proceso de adaptación a esta.

Mercado y Hernández (13), en su estudio cualitativo, describen que entre las manifestaciones y trastornos emocionales derivados de los padecimientos que presentan las personas con enfermedades crónicas destacan la tristeza, la 
desesperación, los deseos de llorar, la irritabilidad, la depresión y su poca tolerancia ante situaciones adversas. Señalan que varios enfermos reconocían los efectos de tales manifestaciones en su vida laboral, en términos de que les imposibilita trabajar, no son los mismos y se vuelven dependientes de quien los cuida 0 ayuda a realizar sus labores.

La familia ejerce un papel crucial en el manejo de los cuidados del paciente enfermo crónico, pues sus limitaciones tendrán un impacto en todo el conjunto familiar, esto modificará la interrelación establecida entre los diferentes miembros.

Tal como señala Pelechano (17), es posible que se generen necesidades de adaptación del domicilio, una mayor carga económica, aislamiento social, modificación de los objetivos de la familia y, como consecuencia de todo esto, cambios a nivel emocional.

\section{AMELOGÉNESIS IMPERFECTA Y SU IMPACTO EMOCIONAL EN LOS PACIENTES}

Parekh et al. (18) señalan que parece sorprendente que aún no se haya estudiado lo suficiente el impacto psicológico de las anomalías dentales.

En la Al la mayor parte del esmalte está afectado en todos los dientes, no alcanza la espesura normal, ocurriendo una variación considerable en los aspectos clínicos. Esta anomalía produce un gran efecto negativo en la estética, debido a sus características clínicas (19).

Sneller et al. (20) reportan en su artículo que el impacto de los defectos visibles afecta la salud psicológica de manera importante, imponiendo retos psicosociales de interacción e incluso una autopercepción negativa.
Según Welbury y Shaw (21), los niños diagnosticados con defectos del esmalte con características estéticas similares a la $\mathrm{Al}$, experimentaron constantes burlas.

Uno de los principales aspectos psicológicos de las enfermedades crónicas es el sufrimiento, este es, quizá, el elemento más importante en los pacientes que las experimentan, pues los acompaña durante toda la vida, y si no reciben el manejo adecuado, puede llegar a afectar en gran parte al individuo y a su padecimiento.

El sufrimiento, según Montalvo et al. (22), se puede definir como un malestar provocado por la amenaza a la integridad de una persona, por lo tanto es una experiencia que abarca todos los elementos que componen la existencia de una persona y que se evidencia de forma individual, es una experiencia subjetiva vivenciada solo por quien lo padece.

Montalvo et al. (22) definen el sufrimiento como «un complejo estado afectivo, cognitivo y negativo que se caracteriza por la sensación de amenaza a la integridad, un sentimiento de impotencia para hacer frente a dicha amenaza, y por el agotamiento de los recursos personales que permiten enfrentarla». Múltiples aspectos pueden ser los causantes del sufrimiento, entre los cuales se encuentran los físicos, psicológicos, socioculturales, espirituales y existenciales. Sus componentes pueden ser aditivos, es decir, que algún factor causal exacerbe otros aspectos del sufrimiento total en el paciente y su familia. A pesar de esto, los componentes se consideran independientes uno del otro.

Algunos factores que contribuyen al sufrimiento son la pérdida de autonomía, la baja autoestima y la desesperanza, así como aspectos 
relacionados con la dignidad y la autoimagen, el aislamiento social, la culpa, entre otros, pues este está condicionado por determinantes sociales y culturales (22).

Es común relacionar dolor y sufrimiento, sin embargo no son sinónimos. Aunque el sufrimiento puede tener su origen en el dolor físico, también puede haber sufrimiento sin dolor. Por otra parte, el dolor puede ser vivido sin sufrimiento (22).

El padecimiento de enfermedades o condiciones tales como la Al provoca sufrimiento en quienes lo padecen, pues tiene repercusiones sociales y emocionales desde la niñez (23).

Tal como indica Gómez (24), el significado que la persona le da a la enfermedad influye directamente en sus reacciones emocionales y de comportamiento. La experiencia de una enfermedad no es completamente negativa, muchos pacientes logran el éxito en la adaptación a los cambios impuestos por esta.

Ante esto, la relación de la persona, que padece la condición, con su familia o personas que lo rodean en su entorno es de gran relevancia, pues ayuda mucho al desarrollo en las diferentes esferas de su vida. El apoyo es esencial para el crecimiento, tanto emocional como psicosocial.

La Al se relaciona principalmente con niños y adolescentes por presentarse desde la erupción de los dientes, por lo tanto, es necesario entender la enfermedad crónica en el adolescente.

Iglesias (25) indica que la enfermedad crónica altera el desarrollo normal biopsicosocial del adolescente, aunque muchos de los padecimientos son leves, otros pueden alterar, gravemente, la calidad de vida de quien lo sufre. La enfermedad crónica a veces es una enfermedad extraña, lo que provoca aislamiento familiar ante la falta de experiencia y conocimiento de los profesionales para su tratamiento. Además, muchas enfermedades y sus tratamientos son dolorosos, con lo que se incrementa la carga de estrés.

Asimismo, se interrumpen las actividades escolares y sociales con frecuencia, por motivos de tratamiento, lo que causa un desajuste con sus pares y el desarrollo educativo. La enfermedad se vuelve omnipresente en la vida cotidiana del niño y adolescente, lo cual crea estrés adicional y más cargas que a las familias de los sanos que no la presentan (25).

Las enfermedades crónicas pueden interferir en el desarrollo físico y psicosocial del adolescente en los aspectos de relación con sus compañeros, físicos e identidad, razón por la cual se debe brindar una atención integral temprana (25).

Cuando un paciente joven es afectado por algún problema dental como la Al se altera todo su entorno psicosocial, ya que el individuo va a presentar problemas de socialización. Las consecuencias implican deterioro en la autoimagen, la autoestima y sentimientos de inseguridad (26). El impacto biopsicosocial de la Al es tan marcado que hace que el tratamiento dental sea una necesidad médica por incluir en las coberturas de los seguros médicos (11). La salud dental se relaciona con indicadores de calidad de vida y está siendo ampliamente usada para medir el impacto de las condiciones bucales de la sociedad (27).

Es importante considerar que los adolescentes representan un grupo desafiante en términos de salud bucal porque tienen dientes permanentes vulnerables en un momento en que establecen su independencia respecto a los padres. Está demostrado que se necesita animar a este grupo para mejorar su salud (28).

La restauración de los defectos de esmalte es importante no solo debido a las preocupaciones 
estéticas, funcionales, fonéticas, sino porque pueden representar un impacto psicológico para el paciente. El manejo de los pacientes con Al debe iniciarse desde muy temprana edad para mejorar su calidad (29).

Específicamente, la sonrisa desempeña una función fundamental en la vida de adultos, adolescentes y niños, siendo importante para su bienestar general y emocional. Asimismo, una sonrisa saludable se relaciona con el atractivo físico, que representa un papel significativo en la forma como verse y sentirse y de cómo se es visto por los otros (19).

De acuerdo con la psicología, una sonrisa saludable es un medio por el cual se comienzan a formar relaciones interpersonales y la autoestima. Sin embargo, no siempre todos pueden sonreír como les gustaría, debido a la presencia de anomalías dentales, por ejemplo la Al, que colocan a los pacientes que la padecen en desventaja psicosocial, perjudicando su estética, sonrisa y afectando su calidad de vida (19).

Lo anterior, en los últimos años, ha generado un aumento significativo en el tratamiento estético y de una sonrisa saludable en la consulta odontológica. El odontólogo, debe identificar los pacientes portadores de diversas anomalías dentales y es su responsabilidad realizar el diagnóstico y tratamiento de estas o referirlas a donde corresponda (19).

Calero y Soto (29) señalan que para el tratamiento de pacientes con Al se debe tomar en cuenta factores como la edad, el tipo y el grado de severidad de la afección, la situación intraoral y el estado socioeconómico. En este tipo de paciente se recomienda un estricto control periódico, adecuada higiene oral, dieta pobre en agentes cariogénicos y fluorización frecuente con la intención de fortalecer el esmalte que permanece (30).

\section{METODOLOGÍA}

El presente es un estudio descriptivo. La unidad de estudio correspondió a familias costarricenses con miembros diagnosticados con lesiones de Al y sus familiares.

La unidad informante fueron pacientes identificados con Al en la Facultad de Odontología de la Universidad de Costa Rica. Se entrevistaron los miembros afectados de diferentes familias costarricenses para la identificación de variables en el componente dental y emocional.

Se identificaron familias nuevas referidas por la clínica de Odontopediatría de la Universidad de Costa Rica y la consulta privada.

Se realizó una entrevista personal, no focalizada, en pacientes y familiares; y con base en las variables identificadas, se elaboró un instrumento para recolección de datos (cuestionario), el cual se sometió a una prueba piloto $\mathrm{y}$, posteriormente, se aplicó a diferentes miembros de familias afectados de Al. La muestra para esta etapa la constituyó 18 personas, quienes completaron el cuestionario.

El análisis estadístico de los datos se realizó mediante el programa EPI INFO.

\section{RESULTADOS Y DISCUSIÓN}

En esta etapa del estudio participaron 18 personas que pertenecen a 17 familias costarricenses diagnosticadas clínicamente con la condición de Al.

El registro de los datos se llevó a cabo por medio de un cuestionario, previamente diseñado, en el que incluyeron las variables edad, sexo y preguntas relacionadas con las principales: aspecto emocional y tratamiento dental. 
Se elaboró una prueba piloto del cuestionario con la cual se realizaron las modificaciones al instrumento que se utilizó.

Se estableció un nivel de significancia del $5 \%$ para las pruebas de hipótesis de igualdad de promedios en la edad.

De los pacientes que participaron, 11 fueron mujeres con edad promedio de 26 años (IC95 \%: 18 - 35) y 7 hombres con una edad promedio de 24 años (IC95 \%: 16 -33), sin que se presentara diferencia estadísticamente significativa $(p=0.664)$ por sexo (Tabla 1).

Tabla 1. Distribución de los pacientes según grupo de edad por sexo: 2014

\begin{tabular}{cccc}
\hline & \multicolumn{3}{c}{ Sexo } \\
\hline $\begin{array}{c}\text { Grupo } \\
\text { de edad }\end{array}$ & Total & Masculino & Femenino \\
\hline Total & 18 & 7 & 11 \\
Menos de 20 & 7 & 2 & 5 \\
$20-29$ & 5 & 4 & 1 \\
$30-39$ & 4 & 0 & 4 \\
40 y más & 2 & 1 & 1 \\
\hline
\end{tabular}

Fuente: Gamboa, L.C., López, A.C., Meza, A.M., Morales, F., Murillo, G. (2014).

Como se observa en la figura 1 , los aspectos que más influyen en la vida de los participantes, de acuerdo con las respuestas del cuestionario, son la autoestima (68.8\%), seguido por factor económico (62.5\%) y por las relaciones interpersonales (50.0\%).

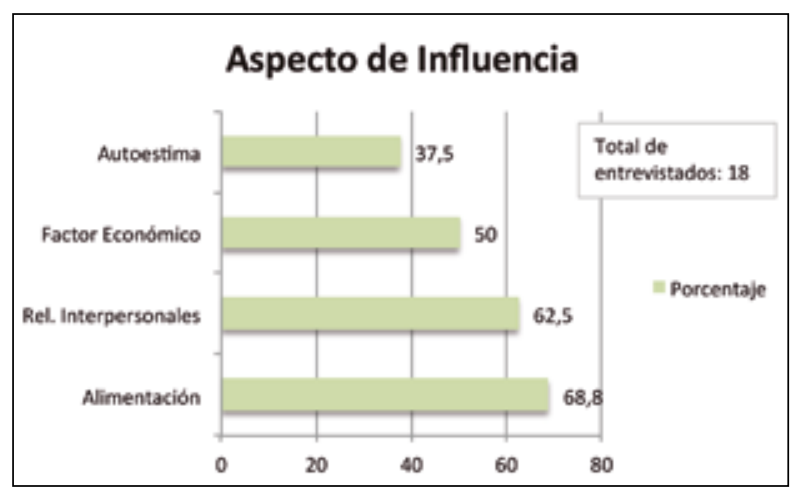

Figura 1. Aspectos influyentes en la vida de los participantes. Fuente: Gamboa, L.C., López, A.C., Meza, A.M., Morales, F., Murillo, G. (2014).

El $89 \%$ considera que los dientes ocupan un lugar importante, más que lo normal en sus vidas, sin que se presente diferencia estadísticamente significativa por sexo $(p=0.732)$.

El 100 \% consideró que el cuidado y atención de sus dientes es algo fundamental en la vida.

Las preocupaciones más importantes vinculadas con su condición de Al se relacionan con la herencia a los hijos (77.8\%), factor económico (66. $7 \%$ ), cuando se debe cubrir el costo del tratamiento dental, seguido por burlas o rechazo social (Figura 2). Siendo la herencia a los hijos, burlas y rechazo social lo que más les ha afectado. Estas familias poseen un conocimiento previo de la condición y saben que, inevitablemente, los hijos van a presentarla, además de enfrentar lo mismo que ellos han pasado. Asimismo, todos los entrevistados fueron pacientes que han pasado por un tratamiento dental largo y costoso para su rehabilitación. 
Las burlas y ridículo social se encuentran en el ámbito de la formación de la personalidad, estos se conocen en salud mental como desestabilizadores cognitivos. En el caso de este estudio, estos desestabilizadores se evidencian en angustia, preocupación, anhedonia 0 desesperanza, ya que casi, irremediablemente, sus hijos padecerán la condición.

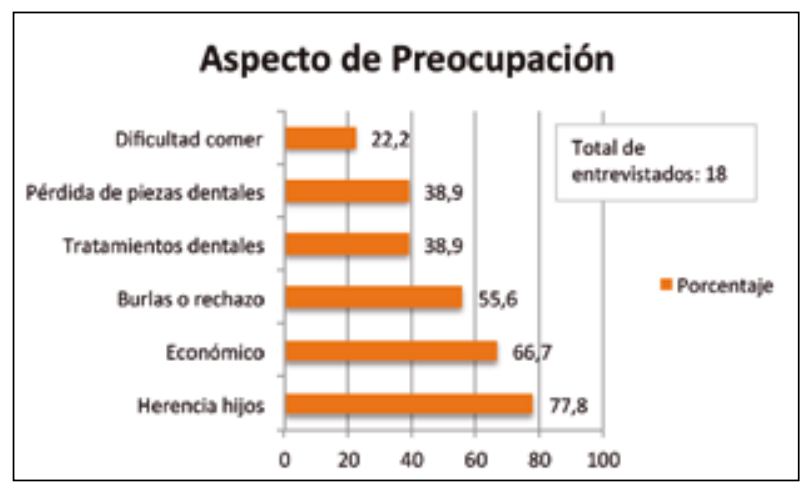

Figura 2. Preocupaciones más importantes a la condición de amelogénesis imperfecta.

Fuente: Gamboa, L.C., López, A.C., Meza, A.M., Morales, F., Murillo, G. (2014).

Esta condición, en donde el área estética es tan importante para relacionarse socialmente y la sonrisa se enmarcan como elementos fundamentales para proyectarse a los demás, se convierten en una situación que agrega al individuo una gran carga emocional de frustración, enojo y tristeza. Esto porque, irremediablemente, al padecerla, la persona se ve lastimada en el área afectiva, relacional, laboral y funcional familiar.

La mitad de los pacientes indicaron que la condición les ha generado alguna obsesión o preocupación extrema, sin embargo, el comportamiento se diferencia por sexo, ya que al $66 \%$ de las mujeres y solo al $25 \%$ de los hombres si les afecta.

Los entrevistados indicaron que, antes de la restauración, percibieron, en apariencia, que sus dientes eran poco estéticos (56\%) y también amarillentos (44\%). En la actualidad, manifestaron que sus dientes los percibían bonitos. Hay que tener en cuenta que todas las personas que participaron en el estudio se restauraron dentalmente 0 se encuentran en el proceso, por lo que los resultados reflejan que, hoy en día, se sienten conformes con la apariencia de sus dientes, debido a las restauraciones.

Con respecto a los tratamientos odontológicos que han recibido, se destaca una amplia gama de ellos: coronas, puentes, carillas, exodoncia, prótesis, resinas y amalgamas, endodoncia, ortodoncia entre otros, siendo las más usuales, como tratamiento definitivo estético, las carillas y coronas. Solamente un participante del estudio es edéntulo total; sin embargo, los resultados señalan que el tratamiento con mayor prevalencia son las extracciones dentales, esto debido a que la mayoría de los participantes han recibido, por lo menos, una exodoncia por diversos motivos, no necesariamente por la condición de Al (Figura 3).

El $45 \%$ de los entrevistados perciben sensibilidad en los dientes, principalmente con ciertos alimentos y, especialmente, los fríos. Este resultado concuerda con las carillas y coronas de porcelana, las cuales son sus restauraciones actuales. 


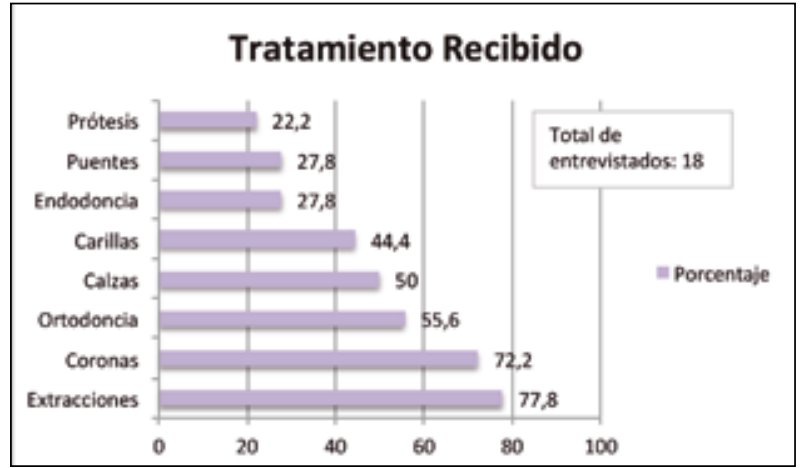

Figura 3. Prevalencia de tratamientos dentales.

Fuente: Gamboa, L.C., López, A.C., Meza, A.M., Morales, F., Murillo, G. (2014).

Al recibir tratamientos dentales, la situación que les genera más molestias cuando acuden al consultorio dental es el aire a presión (70 \%).

El $61 \%$ de los entrevistados consideraron que sus piezas dentales naturales (antes de ser restauradas) eran porosas e irregulares (desde que recuerdan).

Como se describe en la figura 4, los entrevistados describieron la textura de sus dientes con superficies irregulares (55.6\%) y porosas (50.0\%). Estas respuestas se refieren al grupo de participantes que contestaron el cuestionario, los cuales recuerdan bien el curso de su condición dental pasada.

El $55 \%$ indica mantener una higiene dental normal, o sea que no presentan alguna molestia 0 complicación en particular, el 72 \% que su aliento lo consideran normal, un $22 \%$ fresco y solamente una persona respondió que consideraba presentar mal aliento. Todos los demás respondieron que ninguna otra persona les ha hecho notar una observación sobre su aliento. El 40 \% indica que su dentición decidua presentaba una apariencia normal.

Enlaparte final del instrumento de recolección de datos (cuestionario), se proporcionó un espacio de observaciones para que los participantes comentaran, abiertamente, su vivencia respecto

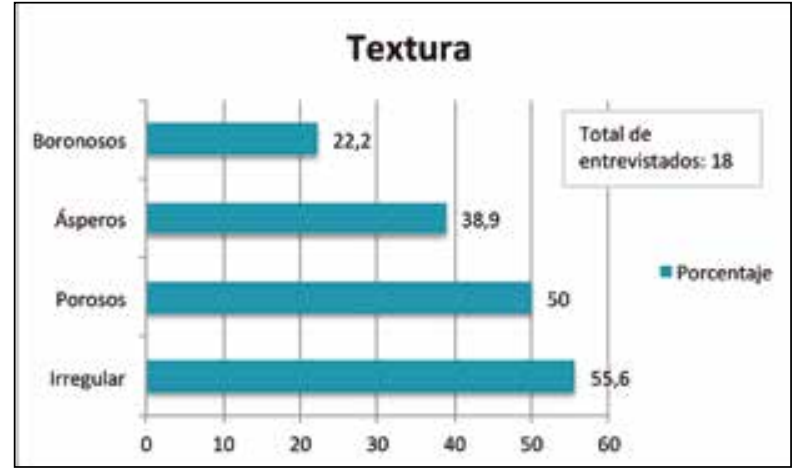

Figura 4. Percepción de la textura de los dientes antes de la restauración. Fuente: Gamboa, L.C., López, A.C., Meza, A.M., Morales, F., Murillo, G. (2014).

de la Al. El $45 \%$ de los encuestados realizó el comentario, un total de 4 hombres y 4 mujeres.

Las personas refieren que los factores más importantes en su experiencia han sido los daños emocionales, entre las que mencionan el marginamiento y la humillación que recibieron a lo largo de su vida. El 85 \% de los colaboradores, en esta parte, relatan que su condición dental les generó problemas de autoestima, en las que se sentían incómodos con su apariencia. Además, al ser una condición de poco conocimiento popular, este generaba preguntas por el «descuido» oral, creando a su alrededor rechazo, humillación y exclusión por la apariencia poco estética.

La discriminación les provocó, en algunos casos, dificultad para encontrar trabajo y espacio para desenvolverse en su ambiente social, ya que su estética no era la adecuada o aceptada para relacionarse abierta y productivamente con otras personas.

A varios de los participantes se les realizó restauraciones dentales, por lo que han logrado un cambio muy significativo en sus vidas en el ámbito personal y social. Refieren que el cambio que sintieron al ver su nueva imagen (sonrisa) les ayudó a superar sus miedos y a ser más seguros para desarrollarse en la sociedad. Este cambio de actitud se respaldó con la reacción de terceras personas hacia ellos, en las que no se recibió más 
maltratos emocionales como los mencionados, provocando que expresen que su vida cambió y actualmente son felices.

Atribuyen esta felicidad a sus odontólogos, mencionando que se encuentran profundamente agradecidos con ellos por haberles brindado comprensión y servicio ante esta condición tan poco conocida por profesionales de la salud.

\section{CONCLUSIONES}

El estado de preocupación extrema en estas personas disminuye sus habilidades para relacionarse de forma exitosa y sentirse aceptadas en su grupo de iguales, demostrarse a sí misma y en el ámbito laboral que son capaces de sobresalir, autovalorarse y poder desarrollar otros talentos a nivel social.

Fue importante la evaluación de la autoestima, ya que es el componente emocional en la estructura de la personalidad. Esta tiene que ver con la capacidad del individuo para sentirse seguro y es el pilar fundamental en el desarrollo de sus decisiones y calidad de afectos proyecte a los demás. La autoestima es la que marcará las herramientas para desarrollar una calidad de vida exitosa o tórpida a lo largo de toda su vida.

Las personas que sufren de Al conocen el valor de una dentición sana, por lo que dan a esta un cuidado especial, tanto visitando al odontólogo con frecuencia como manteniendo buenos hábitos de higiene oral.

La Al conduce a quienes la padecen a pasar por una serie de situaciones que para la mayoría de ellos son comunes, como las burlas y el rechazo social, la inquietud por la herencia a sus hijos, pasar por largos tratamientos que, además de dolorosos, tienen altos costos, por mencionar algunas.

Corresponde al profesional en odontología brindar un abordaje adecuado a esta población, conociendo las situaciones cotidianas a las que se enfrentan, para poder darles una mejor calidad de vida en el ámbito dental y, por ende, en el emocional. Es deber del odontólogo escuchar a estos pacientes, sus quejas y sus experiencias, y realizar adecuados diagnósticos para, posteriormente, realizar tratamientos necesarios que solventen el problema de cada individuo afectado.

\section{REFERENCIAS}

1. Poulter JA, Murillo G, Brookes SJ, Smith CE, Parry DA, Silva S, Kirkham J, Inglehearn CF, Mighell AJ. Deletion of ameloblastin exon 6 is associated with amelogenesis imperfecta. Human Molecuar Genetics. 2014 Oct 15; 23(20):5317-24.

2. Bonilla A, Castro S. Amelogénesis Imperfecta. Revisión de literatura y reporte de un caso. Odontología Vital. 2001. 15, 24-29.

3. Crawford P, Aldred M, Bloch-Zupan A. Amelogenesis imperfecta. Orphanet Journal of Rare Diseases. 2007; 2:17.

4. Varela M, Botella JM, García-Camba JM, García-Hoyos F. Amelogénesis Imperfecta: revisión. Cient Dent. 2008. 5(3); 239-246.

5. Wright JT, Carrion IA, Morris C. The molecular basis of hereditary enamel defects in humans. Journal of Dental Research. 2015; 94(1):52-61.

6. Murillo G, Silva S, Mata M, Esquivel MJ. Amelogénesis Imperfecta. Probabilidad genética de expresión en futuras generaciones de familias costarricenses. ODOVTOS. 2014; 16:71-86. 
7. Gadhia K., McDonald S., Arkutu N., Malik $\mathrm{K}$. Amelogenesis imperfecta: an introduction. British Dental Journal. 2013. 212(8); 377-379.

8. Kamala K., Guddad S. Amelogenesis Imperfecta, an overview. Dept. of Oral Medicine and Radiology. 2012. 5; 84-88.

9. Belmont C., López P. M. Amelogénesis Imperfecta del tipo hipomaduraciónhipoplasia con taurodontismo. Reporte de un caso. División de Estudios de Posgrado e Investigación. UNAM. 1998. 2(8); 18-22.

10. Aldred M. J., Crawford PJM, Savarirayan R. Amelogenesis Imperfecta - a classification and catalogue for the 21st century. Oral Dis. 2003; 9:19-23.

11. Coffield K. D., Phillips C., Brady M., Roberts M. W., Strauss R. P., Wright J. T. The psychosocial impact of developmental dental defects in people with hereditary amelogenesis imperfecta. American Dental Association. 2005 May; 136(5):620-630.

12. Organización Mundial de la Salud. Formulación de estrategias con el fin de alcanzar la salud para todos en el año 2000. Ginebra: OMS; 1994.

13. Mercado F, Hernández E. Las enfermedades crónicas desde la mirada de los enfermos y los profesionales de la salud: un estudio cualitativo en México. Cad. Saúde Pública, Rio de Janeiro. 2007. 23(9); 2178-2186.

14. Vasermanas D. La salud psicológica en las enfermedades crónicas, las repercusiones del estrés. Trabajo presentado en I Jornadas sobre la enfermedad de Behçet. Madrid, España: Asociación española de la enfermedad de Behçet. 2007.

15. Gil-Roales J. Psicología de la salud: aproximación histórica conceptual y aplicaciones. Madrid: Pirámide. 2004.
16. Vinaccia S, Orozco L. Aspectos psicosociales asociados con la calidad de vida de personas con enfermedades crónicas. Diversitas. 2005. 1(2); 125-137.

17. Pelechano V. Calidad de vida, familia y afrontamiento en la enfermedad física crónica: Datos y sugerencias para un modelo. J. Buendia, Familia y Psicología de la Salud. Madrid: Pirámide. 1999.

18. Parekh S, Almehateb M, Cunningham S. How do children with amelogénesis imperfecta feel about their teeth?. International Journal of Paediatric Dentistry. 2014. 24(5); 326-335.

19. Abanto J, Imparato J, Guedes-Pinto A, Bönecke M. Anomalías dentarias de impacto estético en odontopediatría: características y tratamiento. Rev Estomatol Herediana. 2012. 22(3); 171-8.

20. Sneller J, Buchanan H, Parekh S. The impact of amelogénesis imperfecta and support needs of adolescents with $\mathrm{AI}$ and their parents: an exploratory study. International Journal of Pediatric Dentistry. 2014. 24(6); 409-416.

21. Welbury R, Shaw L. A simple technique for removal of mottling, opacities and pigmentation from enamel. Dent Update 1990; 17: 161-163.

22. Montalvo A., Cabrera B., Quiñones S. Enfermedad crónica y sufrimiento: revisión de literatura. Aquichan. 2012. 12(2); 134-143.

23. Craig SA, Baker SR, Rodd HD. How do children view other children who have visible enamel defects?. International Journal of Paediatric Dentistry. 2015; 25(6):399-408.

24. Gómez B. Terapia cognitiva para trastornos psicológicos asociados a enfermedades físicas crónicas. España: Fundación AIGLE. 2011.

25. Iglesias J. L. El adolescente como enfermo crónico. Enfermedad crónica en el adolescente. 
Pediatría Integral. Programa de Formación Continuada en Pediatría Extrahospitalaria. 2008. 19-22.

26. Rodríguez D, Acosta MG, Pierdoménico B, Tortolero MB. La amelogénesis imperfecta tratada por un equipo multidisciplinario. Oral Revista. 2010; 11(33):569-572.

27. Montero J, Bravo M, Albaladejo A, Hernández LA, Rosel EM. Validation the Oral Impact Profile (OHIP-14sp) for adults in Spain. Med Oral Patol Oral Cir Bucal. 2009; 14(1):44-50.
28. Stokes E, Ashcroft A. Plat MJ. Determining Liverpool adolescents' beliefs and attitudes in relation to oral health. Health Educ Res. 2006; 21(2):192-205.

29. Calero JA, Soto L. Amelogénesis Imperfecta. Informe de tres casos en una familia en Cali, Colombia. Colombia Med. 2005. 36(4); 47-50.

30. Vivek R, Singh A, Singh RK, Soni R, Chaturvedi TP. Amelogenesis imperfecta - Functional and esthetic rehabilitation of a mutilated dentition. Indian Journal of Dentistry. 2013; 4(1):60-65. 